\title{
Profile of peginesatide and its potential for the treatment of anemia in adults with chronic kidney disease who are on dialysis
}

This article was published in the following Dove Press journal:

Journal of Blood Medicine

22 May 2012

Number of times this article has been viewed

\section{Ashraf Mikhail}

Renal Unit, Morriston Hospital, Swansea University, Wales, UK
Correspondence: Ashraf Mikhail Renal Unit, Morriston Hospital, Swansea, Wales, UK

Tel +44 I792 7036I9

Email ashraf.mikhail@wales.nhs.uk
Abstract: Peginesatide is a synthetic, dimeric peptide that is covalently linked to polyethylene glycol (PEG). The amino acid sequence of peginesatide is unrelated to that of erythropoietin (EPO) and is not immunologically cross-reactive with EPO. Peginesatide binds to and activates the human EPO receptor, stimulating the proliferation and differentiation of human red cell precursors in vitro in a manner similar to other EPO-stimulating agents (ESAs). In Phase II and III studies in dialysis and predialysis patients, peginesatide administered once monthly was as effective as epoetin alfa given thrice weekly (dialysis patients) or darbepoetin given once weekly (nondialysis patients), in correcting anemia of chronic kidney disease as well as maintaining hemoglobin within the desired target range. In the dialysis population, the reported side-effect profile of peginesatide was comparable to that known with other marketed ESAs. In the nondialysis studies, compared with those treated with darbepoetin, patients treated with peginesatide experienced a higher adverse-effect profile. Peginesatide is likely to be licensed for treatment of renal anemia in dialysis patients and not in nondialysis patients. Despite this limitation, peginesatide is likely to prove valuable in treating dialysis patients because of its infrequent mode of administration, thereby allowing for a reduced number of injections, with associated better compliance, reduced cold storage requirement, and improved stock accountability. PEGylated therapeutic proteins can elicit immunological response to the PEG moiety of the therapeutic complex. Only long-term experience and post-marketing surveillance will address whether this immunological response will have any impact on the clinical efficacy or safety of peginesatide in clinical practice.

Keywords: peginesatide, dialysis, chronic kidney disease

\section{Introduction}

Prior to the introduction of recombinant human erythropoietin (rhuEPO) in clinical practice, many patients receiving dialysis were severely anemic and needed transfusions to maintain a hemoglobin level greater than $7 \mathrm{~g} / \mathrm{dL} .{ }^{1}$ Consequently, patients suffered many of the consequences of chronic anemia, mainly in terms of volume overload, hyperkalemia, iron overload, blood-borne infections, and allosensitization. ${ }^{2}$

The introduction of erythropoiesis-stimulating agents (ESAs) has changed the care of patients with kidney disease by increasing hemoglobin levels and thereby avoiding the need for transfusions. The first ESAs to be introduced were short-acting (eg, epoetin alfa and beta) and required administration three times a week. The introduction of darbepoetin, a second-generation ESA and, more recently, Mircera (Hoffmann-La Roche, Basel, Switzerland), a third-generation ESA (EU only), both with extended duration of action, has led to less-frequent dosing with a comparable efficacy. ${ }^{3}$ All these ESAs 
are derivates of the parent molecule, rhuEPO. The creation of a drug that is structurally different from rhuEPO, yet capable of stimulating erythropoiesis, could be an interesting therapeutic development. The benefit of such a development could be the elimination of the potential to induce immune response to rhuEPO and therefore compromise its action. In its most severe form, such an immune response could manifest as pure red cell aplasia (PRCA), a severe form of anemia unresponsive to all the currently licensed ESAs, rendering affected patients transfusion-dependent. Such a development could also address other unmet needs in treating anemia in chronic kidney disease (CKD) patients.

Peginesatide, a drug capable of stimulating erythropoiesis, and likely to be licensed for clinical use in the near future, is the first ESA that bears no structural similarity to rhuEPO. This mini review will discuss the data available from Phase II and III clinical trials of peginesatide, focusing on its clinical use and safety profile, and will conclude by discussing its potential role in the field of management of anemia of $\mathrm{CKD}$ and possible uncertainties that may be associated with its use in clinical practice.

\section{Peginesatide: structure and preclinical data}

Peginesatide is a synthetic, dimeric peptide that is covalently linked to polyethylene glycol (PEG). Its molecular weight ranges between 45.0 to $50.5 \mathrm{kDa}^{4}{ }^{4}$ The amino acid sequence of peginesatide is unrelated to that of rhuEPO and is not immunologically cross-reactive with rhuEPO. ${ }^{4}$ This characteristic potentially reduces the risk of PRCA, and theoretically may provide a rescue treatment for patients affected by such condition. Peginesatide binds to and activates the human EPO receptor, stimulating the proliferation and differentiation of human red cell precursors in vitro in a manner similar to ESAs. ${ }^{5}$ A predictable, dose-related effect on reticulocyte and hemoglobin levels has been observed in rats and monkeys. ${ }^{6,7}$

\section{Clinical efficacy}

In a Phase I study to evaluate the safety and pharmacodynamic effects of single, intravenous doses $(0.025,0.05$, and $0.1 \mathrm{mg} / \mathrm{kg}$ ) of peginesatide in 28 healthy male volunteers, all doses were well tolerated, with safety profiles comparable to that of placebo. Peginesatide showed a dose-dependent increase in reticulocytes. The $0.1 \mathrm{mg} / \mathrm{kg}$ dose was associated with a statistically significant increase in hemoglobin from baseline compared with the placebo group. That effect was sustained for more than 1 month. ${ }^{4}$

\section{Peginesatide: experience in correcting anemia in CKD patients not on dialysis}

The efficacy of peginesatide in correcting anemia in CKD patients was demonstrated in a Phase II trial. Patients who were not on dialysis, and not receiving treatment with ESAs in the 12 weeks before study drug administration, were sequentially assigned to 1 of 10 cohorts that differed in starting peginesatide dose (different body weight-based or absolute doses), route of administration (intravenous or subcutaneous), and frequency of administration (every 4 or 2 weeks). Across all cohorts, $96 \%$ of patients achieved a hemoglobin response. A dose-response relationship was evident for hemoglobin increase. Comparable subcutaneous and intravenous peginesatide doses produced similar hemoglobin responses. Rapid rates of hemoglobin rise and hemoglobin excursions $>1 \mathrm{~g} / \mathrm{dL}$ tended to occur more frequently with once-fortnightly dosing than with once-monthly dosing. This study provided the first proof that peginesatide administered every 4 weeks can increase and maintain hemoglobin level in nondialysis CKD patients. ${ }^{8}$

Phase III studies have demonstrated that dialysis patients maintained on epoetin alfa can be switched successfully to once-monthly peginesatide (unpublished data).

In CKD patients not on dialysis, although once-monthly peginesatide was noninferior to darbepoetin alfa in both correction of anemia and maintenance of hemoglobin within target range, higher proportions of adverse events and serious adverse events were observed in the peginesatide treatment group compared with the darbepoetin treatment group (unpublished data). The cause of such increased incidence in the peginesatide group compared with the darbepoetin group is unclear. But based on these results, peginesatide is likely to receive licensing approval only for the treatment of anemia in dialysis patients and not for CKD patients not on dialysis.

\section{Correction of anemia in patients with pure red cell aplasia}

Because anti-EPO antibodies have not been observed to cross-react with peginesatide, ${ }^{9,10}$ peginesatide was studied as a potential treatment in CKD patients with anti-EPO antibody-mediated PRCA.

In a Phase II, open-label, multiple-dose study to evaluate the ability of peginesatide administered monthly to increase and maintain hemoglobin levels in these transfusion-dependent patients, 14 patients were treated with peginesatide for a median of 28 months. The median hemoglobin concentration increased from $9.0 \mathrm{~g} / \mathrm{dL}$ 
(with transfusion support in 12 out of 14 patients) before treatment, to $11.4 \mathrm{~g} / \mathrm{dL}$ at the time of the last administration of peginesatide; transfusion requirements diminished within 12 weeks after the first dose, after which 13 of the 14 patients no longer required regular transfusions. ${ }^{11}$

The level of anti-EPO antibodies declined over the course of the study and became undetectable in six patients. One patient who initially responded to treatment had a diminished hematologic response a few months later despite increased doses of peginesatide and required transfusions again; this patient was found to have antibodies against peginesatide. $^{11}$

\section{Antibody responses and clinical effects in clinical studies}

Peginesatide differs from ESAs derived from human EPO in that it is a synthetic, dimeric peptide that does not share structural homology with human EPO. Among 139 patients enrolled in a Phase II study, two patients (1.4\%) had detectable levels of peginesatide-specific neutralizing antibodies. Those two patients showed no evidence of de novo anti-EPO antibodies, and no new cases of PRCA were reported in any patient receiving peginesatide. ${ }^{8}$

\section{Peginesatide: a role in the management of anemia in dialysis patients?}

It is now over 20 years since rhuEPO was first used to treat anemic CKD patients. During this period, the market was dominated with recombinant human EPO or its derivatives. The imminent introduction of peginesatide to the anemia market provides an exciting opportunity to physicians and clinical researchers alike to use a drug that is structurally unrelated to EPO, yet capable of producing its desired therapeutic effects. There are several potential advantages to using an EPO mimetic agent to treat anemic CKD patients.

\section{Peginesatide: a PEGylated EPO mimetic dipeptide}

The conjugation of small proteins with PEG, or PEGylation, has become an increasingly common method of improving the half-life of biological products, mainly through reducing the urinary excretion of the molecule ${ }^{12}$ but also by reducing the enzymic degradation due to the increased steric bulk. ${ }^{1}$ In addition, PEGylated biological products often exhibit a reduced affinity for the target receptor compared with the native precursor. This reduced affinity can lead to a lower elimination by target-mediated clearance mechanisms, and prolonged half-life of the PEGylated product. Finally, the addition of the PEG moiety can have beneficial effects on the immunological profile of a molecule by reducing the ability of the compound to raise antibodies in humans. ${ }^{13}$ All these characteristics make the development of peginesatide an attractive therapeutic alternative to other ESAs in the treatment of anemia in patients with CKD.

\section{A novel peptide}

Being structurally different from other licensed ESAs, peginesatide may be ideally used whenever other ESAs cannot be used (eg, intolerance to therapeutic proteins, nonneutralizing anti-EPO antibodies). Although not within its current license application, its use as a rescue therapy in patients with EPO-induced red cell aplasia may provide a marketing leverage over other available ESAs.

\section{A long-acting preparation}

The use of a long-acting ESA in treating anemia in dialysis patients provides a unique opportunity to create maximum value for patients and healthcare providers alike by reducing waste through improved quality, efficiency, and safety. A long-acting ESA may have the following potentials:

- It may offer more flexibility in ESA dosing and administration compared with the existing shorter-acting preparations.

- It may release more time for health workers to treat more patients and attend to more complicated cases.

- Monthly administration would allow hospital pharmacies to request the drug when needed, with little need for storage.

- The reduced number of injections (from 100-150 injections per patient year to 12 injections per patient year) may translate into a reduced need for cold storage capacity, reduced risk and asset-value loss in the case of cold storage interruption, reduced wastage, and reduced number of missed doses.

- Once-monthly dosing may help address clinical governance issues by facilitating accurate stock, prescription, and administration reconciliation.

\section{The use of peginesatide may provide the opportunity to reduce overall patient exposure to ESA}

Preliminary post hoc analysis of Phase III studies of peginesatide in dialysis patients suggests that for patients receiving high epoetin doses at baseline (ie, prior to switch to 
peginesatide), the relative dose of peginesatide is significantly lower than that for those receiving low epoetin doses at baseline (unpublished data). This suggests that, for patients requiring a high maintenance dose of epoetin, switching to peginesatide may provide the opportunity to reduce overall drug exposure without compromising therapeutic efficacy. Whether this may translate into a better outcome for patients may need further long-term trials to determine.

\section{Peginesatide: residual uncertainties}

Before peginesatide establishes itself as a therapeutic alternative to other licensed ESAs, it has to overcome a few challenges.

\section{The position of ESA therapy in the current anemia market}

The results of two large multicenter studies have suggested a possible association between high hemoglobin level and increased cardiovascular events in patients with CKD. ${ }^{14,15}$ In addition, post hoc analyses of these studies suggests a direct association between ESA dose and survival in CKD patients. ${ }^{16,17}$ Becoming increasingly aware of these data, clinicians are actively trying to minimize ESA utilization, and to rely alternatively on parenteral iron therapy. This is compounded by the fact that national and international guidelines on anemia management have brought therapeutic target hemoglobin in ESA-treated patients to a lower level in response to the results of these multicenter studies. ${ }^{18,19}$

\section{Price}

As more ESAs are introduced into the ever-expanding anemia market, the marketing price of ESA products is being reduced by the pharmaceutical industry to maintain their position in the anemia market. This is particularly relevant in Europe, where several biosimilars have been in the market for over 4 years now. This is also important given the current financial climate where healthcare providers are continually exploring new avenues to make health service delivery more affordable and cost-effective. Peginesatide has to be marketed at a price that allows it to compete with other well-established ESAs.

\section{Restricted license}

The drug is likely to be licensed for the treatment of renal anemia in chronic dialysis patients only. Based on the available safety data, Affymax (Palo Alto, CA) is not seeking marketing authorization for the treatment of renal anemia in patients not on dialysis. Given the fact that the majority of dialysis patients start ESA therapy before they start dialysis, patients may need to switch to peginesatide as they commence renal replacement therapy. A peginesatide marketing campaign may need to establish a strong reason to convince stakeholders of the benefits of switching patients as they commence renal replacement therapy.

\section{Peginesatide: just another ESA?}

Although structurally different from rhuEPO, peginesatide acts in a mechanism similar to other ESAs available for clinical use (ie, via stimulation of EPO receptors), has to be administered parenterally, and possibly shares the same safety profile as other ESAs. Clinicians will ask for a convincing case to switch patients from existing ESAs that have been in clinical practice for over a decade, with well over one million patient/year exposure and a well-documented safety profile, ${ }^{20}$ to the newly introduced peginesatide, with comparatively limited safety data and relatively limited information on clinical experience.

\section{Compliance}

With the use of long-acting ESA in treating chronic dialysis patients, there is a need to ensure a maximum compliance with ESA administration. In a cross-sectional study on UK dialysis patients, concordance (defined as receiving $\geq 90 \%$ of the prescribed dose) ranged from $24 \%$ to $33 \% .{ }^{21}$ While missing a single injection of a short-acting ESA preparation may result in a transient reduction of the overall area under the curve of ESA blood level, missing a whole monthly ESA injection may translate into a more significant and protracted drop in the area under the curve, with possible consequences on hemoglobin levels. Therefore, for units contemplating the use of monthly ESA preparation for their dialysis preparation, a robust drug accountability/risk-management plan should be established to minimize the risk of missed dose, or of over- or underdosing. Nevertheless, it is also important here to note that the above study found that reduced frequency of administration was associated with fewer missed doses.

\section{Metabolic fate of PEGylated ESA in CKD patients}

The clearance via metabolism of the PEGs that are typically used to alter the pharmacokinetics of biological products (molecular weight of 5000 or greater) $)^{22,23}$ is likely to be insignificant. This is because the metabolism of PEG is molecular weight-dependent, with high-molecular-weight PEGs $(>4000)$ showing less metabolism. ${ }^{24}$ The major route of elimination of PEG occurs through passive glomerular 
filtration and is dependent on molecular weight. ${ }^{24}$ Human excretion balance studies have shown that $86 \%$ and $96 \%$ of PEG 1000 and 6000 were excreted in the urine 12 hours after intravenous administration. ${ }^{25,26}$ These data raise questions about the metabolic fate of peginesatide as well as other PEGylated ESAs in patients with CKD, particularly in dialysis patients, many of whom have a markedly diminished or even zero glomerular filtration rate. In these patients, PEG is likely to accumulate in tissues in patients who receive long-term treatment with the drug. In fact, there are no systematic long-term studies that show: (1) whether PEG is excreted completely or partly remains in the body, (2) where it is accumulated, and (3) its effects at the sites of accumulation. ${ }^{27}$ Ideally, the fate of any pharmaceutical product should be addressed via relevant pharmacokinetic studies, but this is impossible to achieve with PEGylated proteins in clinical practice. This is due to several reasons; first, identifying metabolites of PEG will be difficult because the PEGs used always have polydispersed molecular weight. ${ }^{22}$ Second, subtle changes in mass due to metabolism will be undetectable by mass spectrometry because of the range of molecular weights present in the PEG. Also, it has been shown that PEG can suppress ionization in a mass spectrometer. ${ }^{28}$ In addition, humans and animals are commonly exposed to PEG via a variety of environmental sources, which means that it is likely that human plasma, urine, and other body fluids contain a range of PEGs and PEG metabolites. When the trace doses of most PEGylated biological products are combined with the ubiquitous "contamination" likely to be seen in animals and humans, it is unlikely that the approach to metabolite identification would be successful. ${ }^{27}$ Therefore, only post-marketing long-term surveillance will be able to address the long-term effects of the use of PEGylated ESAs in patients with $\mathrm{CKD}$.

\section{PEGylated ESA: a possible immune reaction?}

It has been shown that PEG, which is not supposed to show any opsonization, can in fact induce specific as well as nonspecific recognition by the immune system, thereby leading to a response of the body to intravenously administered PEG formulations.

\section{Anti-PEG antibodies}

Animal studies have clearly shown that some PEGylated proteins can elicit antibody formation against PEG. ${ }^{29-31}$ This anti-PEG response can accelerate the clearance of PEGylated proteins. ${ }^{32-34}$ In humans, PEG antibodies can be produced and may limit therapeutic efficacy, and may even reduce tolerance of PEG-asparaginase in patients with acute lymphoblastic leukemia, ${ }^{35}$ and of pegloticase in patients with chronic gout. ${ }^{36,37}$ Of major importance is the recent finding of a $22 \%$ to $25 \%$ occurrence of $P E G$ antibodies in 350 healthy blood donors. ${ }^{35,38}$ It has been suggested that this increase is most likely due to greater exposure to PEG and PEG-containing compounds in cosmetics, pharmaceuticals, and processed food products. ${ }^{35}$ These authors recommend that patients should be screened for pre-existing anti-PEG and monitored for the development of anti-PEG throughout the course of treatment with any PEG containing agent. ${ }^{35}$ It is not clear whether this immunological response to PEG may have contributed to the unfavorable hematological response observed among nondialysis CKD patients treated with peginesatide compared with those treated with darbepoetin in Phase III studies. In addition, the available clinical data on peginesatide did not evaluate the prevalence of anti-PEG antibody response among patients treated with peginesatide. It is hoped that this aspect will be addressed in the upcoming publication of the results of Phase III clinical trials of peginesatide in patients with CKD.

\section{PEG-induced complement activation}

It has been shown that PEG, administered at doses equivalent to those used to bind therapeutic proteins, can generate complement activation products in human serum on a time scale of minutes via activation of the alternative complement pathway. PEG-induced complement activation has been reported to cause anaphylaxis. ${ }^{39,40}$ An immediate hypersensitivity reaction (HSR) in 5\% to $10 \%$ of treated patients was shown for different PEG-containing liposomal carriers. ${ }^{27}$ Complement activation with subsequent HSR was demonstrated with $99 \mathrm{mTc}$-labeled $2 \mathrm{kDa}$ methoxyPEG-liposomes for the treatment of Crohn's disease. ${ }^{41}$ The PEGylated liposome formulation of doxorubicin used in anticancer therapy also caused HSR in up to $25 \%$ of patients, despite pretreatment with corticosteroids and antihistamines, and without prior sensitization. ${ }^{39}$ This PEG-induced effect may be of particular relevance among some groups of CKD patients who are known to have impaired alternative complement activation as a possible mechanism of initiation and progression of their kidney disease, such as patients with mesangiocapillary glomerulonephritis/dense deposit disease or certain forms of hemolytic uremic syndrome.

\section{Conclusion}

Peginesatide is a novel peptide with no structural similarity to rhuEPO, yet capable of stimulating erythropoiesis in patients 
with anemia of CKD. It is likely to gain a license for treating anemia in CKD patients who are on dialysis. Peginesatide may also be used as a rescue therapy in patients who develop EPOinduced PRCA. Once licensed, long-term clinical experience may provide insights on its potential as an ESA-minimizing agent in patients requiring large maintenance ESA doses. Peginesatide will probably have the same hematological safety profile as other available ESAs. PEG present in the therapeutic PEGylated proteins may contribute to the adverse-event profile, probably through activation of anti-PEG antibodies and complement consumption via the alternative pathway. Only long-term clinical experience/pharmacovigilance data will determine the exact place of peginesatide in the field of anemia management in patients with CKD.

\section{Disclosure}

Dr Ashraf Mikhail wishes to declare the following conflicts of interest: study investigator for research studies sponsored by Amgen, Roche, Affymax, and Takeda; receipt of sponsorship to attend scientific meetings from Amgen, Roche, and Johnson \& Johnson; consultancy fees from Amgen, Roche, Astellas, Takeda, and Lipoxen.

\section{References:}

1. Lankhorst CE, Wish JB. Anemia in renal disease: diagnosis and management. Blood Rev. 2009;24(1):39-47.

2. Lemy A, Andrien M, Wissing KM, et al. Major histocompatibility complex class 1 chain-related antigen antibodies: sensitizing events and impact on renal graft outcomes. Transplantation. 2010;90(2):168-174.

3. Lankhorst CE, Wish JB. Anemia in renal disease: diagnosis and management. Blood Rev. 2009;24(1):39-47.

4. Stead RB, Lambert J, Wessels D, et al. Evaluation of the safety and pharmacodynamics of Hematide, a novel erythropoietic agent, in a phase 1, double-blind, placebo-controlled, dose-escalation study in healthy volunteers. Blood. 2006;108(6):1830-1834.

5. Green JM, Pan Y. Hematide ${ }^{\mathrm{TM}} /$ Peginesatide binds to the human erythropoietin receptor with high affinity and favorable thermodynamics. Poster presentation ASN Renal WeekEnds. JASN 21;2010:165A. Available from: http://www.ASN-online.org/. Accessed November 5, 2011.

6. Woodburn KW, Wilson SD, Fong KL, et al. Chronic preclinical safety evaluation of Hematide, a pegylated peptidic erythropoiesis stimulating agent in monkeys. Haematologica. 2008;93(9):1376-1379.

7. Woodburn KW, Schatz PJ, Fong KL, et al. Preclinical safety and pharmacology of Hematide, a peptidic erythropoiesis stimulating agent (ESA), in rats and monkeys. Drug Chem Toxicol. 2008;31(2): 229-244.

8. Macdougall IC, Wiecek A, Tucker B, et al. Dose-finding study of peginesatide for anemia correction in chronic kidney disease patients. Clin J Am Soc Nephrol. 2011;6(11):2579-2586.

9. Woodburn KW, Fan Q, Winslow S, et al. Hematide is immunologically distinct from erythropoietin and corrects anemia induced by antierythropoietin antibodies in a rat pure red cell aplasia model. Exp Hematol. 2007;35(8):1201-1208.

10. Woodburn KW, Schatz PJ, Fong KL, Beaumier P. Erythropoiesis equivalence, pharmacokinetics and immune response following repeat hematide administration in cynomolgus monkeys. Int J Immunopathol Pharmacol. 2010;23(1):121-129.
11. Macdougall IC, Rossert J, Casadevall N, et al. A peptide-based erythropoietin-receptor agonist for pure red-cell aplasia. $N$ Engl J Med. 2009;361(19):1848-1855.

12. Yang BB, Lum PK, Hayashi MM, Roskos LK. Polyethylene glycol modification of filgrastim results in decreased renal clearance of the protein in rats. J Pharm Sci. 2004;93(5):1367-1373.

13. Mehvar R. Modulation of the pharmacokinetics and pharmacodynamics of proteins by polyethylene glycol conjugation. J Pharm Pharm Sci. 2000;3(1):125-136.

14. Pfeffer MA, Burdmann EA, Chen CY, et al. A trial of darbepoetin alfa in type 2 diabetes and chronic kidney disease. NEngl J Med. 2009;361(21): 2019-2032.

15. Singh AK, Szczech L, Tang KL, et al. Correction of anemia with epoetin alfa in chronic kidney disease. N Engl J Med. 2006;355(20): 2085-2098.

16. Szczech LA, Barnhart HX, Sapp S, et al. A secondary analysis of the CHOIR trial shows that comorbid conditions differentially affect outcomes during anemia treatment. Kidney Int. 2010;77(3): 239-246.

17. Szczech LA, Barnhart HX, Inrig JK, et al. Secondary analysis of the CHOIR trial epoetin-alpha dose and achieved hemoglobin outcomes. Kidney Int. 2008;74(6):791-798.

18. KDOQI; National Kidney Foundation. KDOQI Clinical practice guidelines and clinical practice recommendations for anemia in chronic kidney disease. Am J Kidney Dis. 2006;47(5 Suppl 3):S11-S145.

19. Mikhail A, Shrivastava R, Richardson D. Renal Association Clinical Practice Guideline on anaemia of chronic kidney disease. Nephron Clin Pract. 2011;118 Suppl 1:c101-c124.

20. Bennett CL, Cournoyer D, Carson KR, et al. Long-term outcome of individuals with pure red cell aplasia and antierythropoietin antibodies in patients treated with recombinant epoetin: a follow-up report from the Research on Adverse Drug Events and Reports (RADAR) Project. Blood. 2005;106(10):3343-3347.

21. Mahon A, Docherty B. Renal anaemia - the patient experience. EDTNA-ERCA J. 2004;30(1):34-37.

22. Veronese FM, Pasut G. PEGylation, successful approach to drug delivery. Drug Discov Today. 2005;10(21):1451-1458.

23. Molineux G. Pegylation: engineering improved biopharmaceuticals for oncology. Pharmacotherapy. 2003;23(8 Pt 2):3S-8S.

24. Webster R, Didier E, Harris P, et al. PEGylated proteins: evaluation of their safety in the absence of definitive metabolism studies. Drug Metab Dispos. 2007;35(1):9-16.

25. Shaffer CB, Critchfield FH. The absorption and excretion of the solid polyethylene glycols; (carbowax compounds). J Am Pharm Assoc Am Pharm Assoc. 1947;36(5):152-157.

26. Shaffer CB, Critchfield FH, Nair JH 3rd. The absorption and excretion of a liquid polyethylene glycol. J Am Pharm Assoc Am Pharm Assoc. 1950;39(6):340-344.

27. Knop K, Hoogenboom R, Fischer D, Schubert US. Poly(ethylene glycol) in drug delivery: pros and cons as well as potential alternatives. Angew Chem Int Ed Engl. 2010;49(36):6288-6308.

28. Weaver R, Riley RJ. Identification and reduction of ion suppression effects on pharmacokinetic parameters by polyethylene glycol 400 . Rapid Commun Mass Spectrom. 2006;20(17):2559-2564.

29. Richter AW, Akerblom E. Antibodies against polyethylene glycol produced in animals by immunization with monomethoxy polyethylene glycol modified proteins. Int Arch Allergy Appl Immunol. 1983;70(2): 124-131.

30. Tsuji J, Hirose K, Kasahara E, Naitoh M, Yamamoto I. Studies on antigenicity of the polyethylene glycol (PEG)-modified uricase. Int J Immunopharmacol. 1985;7(5):725-730.

31. Sroda K, Rydlewski J, Langner M, Kozubek A, Grzybek M, Sikorski AF. Repeated injections of PEG-PE liposomes generate anti-PEG antibodies. Cell Mol Biol Lett. 2005;10(1):37-47.

32. Cheng TL, Wu PY, Wu MF, Chern JW, Roffler SR. Accelerated clearance of polyethylene glycol-modified proteins by anti-polyethylene glycol IgM. Bioconjug Chem. 1999;10(3):520-528. 
33. Cheng TL, Chen BM, Chern JW, Wu MF, Roffler SR. Efficient clearance of poly(ethylene glycol)-modified immunoenzyme with anti-PEG monoclonal antibody for prodrug cancer therapy. Bioconjug Chem. 2000;11(2):258-266.

34. Wang XY, Ishida T, Kiwada H. Anti-PEG IgM elicited by injection of liposomes is involved in the enhanced blood clearance of a subsequent dose of PEGylated liposomes. J Control Release. 2007;119(2):236-244.

35. Armstrong JK, Hempel G, Koling S, et al. Antibody against poly(ethylene glycol) adversely affects PEG-asparaginase therapy in acute lymphoblastic leukemia patients. Cancer. 2007;110(1):103-111.

36. Reinders MK, Jansen TL. New advances in the treatment of gout: review of pegloticase. Ther Clin Risk Manag. 2010;6:543-550.

37. Burns CM, Wortmann RL. Gout therapeutics: new drugs for an old disease. Lancet. 2011;377(9760):165-177.
38. Garratty G. Modulating the red cell membrane to produce universal/ stealth donor red cells suitable for transfusion. VoxSang. 2008;94(2): 87-95.

39. Chanan-Khan A, Szebeni J, Savay S, et al. Complement activation following first exposure to pegylated liposomal doxorubicin (Doxil): possible role in hypersensitivity reactions. Ann Oncol. 2003;14(9): 1430-1437.

40. Szebeni J. Complement activation-related pseudoallergy: a new class of drug-induced acute immune toxicity. Toxicology. 2005;216(2-3): $106-121$.

41. Brouwers AH, De Jong DJ, Dams ET, et al. Tc-99m-PEG-Liposomes for the evaluation of colitis in Crohn's disease. J Drug Target. 2000; $8(4): 225-233$

\section{Publish your work in this journal}

The Journal of Blood Medicine is an international, peer-reviewed, open access, online journal publishing laboratory, experimental and clinical aspects of all topics pertaining to blood based medicine including but not limited to: Transfusion Medicine; Blood collection, Donor issues, Transmittable diseases, and Blood banking logistics; Immunohematology; Artificial and alternative

\section{Dovepress}

blood based therapeutics; Hematology; Biotechnology/nanotechnology of blood related medicine; Legal aspects of blood medicine; Historical perspectives. The manuscript management system is completely online and includes a very quick and fair peer-review system. Visit http://www.dovepress.com/ testimonials.php to read real quotes from published authors.

Submit your manuscript here: http://www.dovepress.com/Journal-of-blood-medicine-journal 